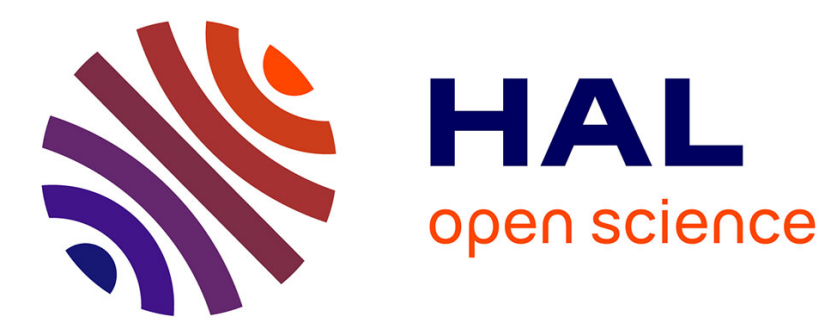

\title{
Second-Order Complex Random Vectors and Normal Distributions
}

Bernard Picinbono

\section{To cite this version:}

Bernard Picinbono. Second-Order Complex Random Vectors and Normal Distributions. IEEE Transactions on Signal Processing, 1996, 44, pp.2637 - 2640. hal-01736682

\section{HAL Id: hal-01736682 \\ https://hal.science/hal-01736682}

Submitted on 18 Mar 2018

HAL is a multi-disciplinary open access archive for the deposit and dissemination of scientific research documents, whether they are published or not. The documents may come from teaching and research institutions in France or abroad, or from public or private research centers.
L'archive ouverte pluridisciplinaire HAL, est destinée au dépôt et à la diffusion de documents scientifiques de niveau recherche, publiés ou non, émanant des établissements d'enseignement et de recherche français ou étrangers, des laboratoires publics ou privés. 


\title{
Second-Order Complex Random Vectors and Normal Distributions
}

\author{
Bernard Picinbono, Fellow, IEEE
}

\section{The pdf copy of the final published text can be obtained from the author at the folowing address : bernard.picinbono@1ss.supelec.fr}

\author{
Reference of this text : IEEE Trans. on Signal Processing, Vol. 44, No 10, pp. 2637-2640, \\ October 1996.
}

\begin{abstract}
Complex random vectors are usually described by their covariance matrix. This is insufficient for a complete description of second-order statistics, and another matrix called relation matrix is necessary. Some of its properties are analyzed and used to express the probability density function of normal complex vectors. Various consequences are presented.
\end{abstract}

S

\section{INTRODUCTION}

Complex random vectors (RVs) are widely used in many areas of signal processing such as spectral analysis [I] and array processing [2]. However, the statistical properties of RVs effectively used are

Manuscript received

B. P. is with the Laboratoire des Signaux et Systèmes (L2S), a joint laboratory of the C.N.R.S. and the École Supérieure d'Électricité, Plateau de Moulon, 3 rue Joliot-Curie 91192, Gif sur Yvette, France. The L2S is associated with the University of Paris-Sud, France. E-mail: bernard.picinbono@1ss.supelec.fr, . 
essentially limited to those of the covariance matrix. Linear prediction procedures and autoregressive modeling also use only properties of the correlation function of complex signals [1] and [3]. Many questions concerning statistical properties of RVs remain open, however, and some of them will be analyzed in this correspondence. In the first part, we show that the covariance matrix is insufficient to completely describe the statistics of complex RVs, and for this purpose, another matrix is necessary. Its definition and the conditions of its existence are analyzed. By using this matrix, we present the structure of the probability density function (PDF) of normal complex RVs. From this PDF, we deduce the characteristic function and various properties of complex normal random variables. For example, it is shown that contrary to the real case, noncorrelated normal random variables arc not generally independent. Conditional PDFs are also analyzed, and the consequences in mean square estimation are presented.

Let us first remind that a complex $\mathrm{RV} \mathbf{Z}$ of $\mathbb{C}^{n}$ is simply a pair of real $\mathrm{RV}$ s of $\mathbb{R}^{n}$ such that $\mathbf{Z}=\mathbf{X}+j \mathbf{Y}$. It is therefore always possible to treat all the problems concerning complex RVs by using a real RV of $\mathbb{R}^{n}$. However, this procedure is often much more tedious than using directly the RV $\mathbf{Z}$ of $\mathbb{C}^{n}$.

\section{SECOND-ORdER PROPERTIES}

Even if the most interesting second-order properties are related to the covariance matrix $\boldsymbol{\Gamma}$, it does not completely describe the secondorder statistical properties of $\mathbf{Z}$. For this, another matrix $\mathbf{C}$, which we refer to as the relation matrix, is necessary. For zero-mean RVs, these matrices arc defined by

$$
\mathbf{\Gamma} \triangleq E\left(\mathbf{Z Z}^{H}\right) ; \mathbf{C} \triangleq E\left(\mathbf{C C}^{T}\right) .
$$

In these equations, $T$ means transposition, and $H$ means transposition and complex conjugation. The matrix $\boldsymbol{\Gamma}$ is complex, Hermitian, and nonnegative definite (NND). We assume in the following that there is no zero eigenvalue. The matrix $\mathbf{C}$ is complex and symmetric and therefore satisfies $\mathbf{C}^{*}=\mathbf{C}^{H}$. where the star means the complex conjugate. This matrix $\mathbf{C}$ is very rarely introduced in signal processing literature, and the main reason for this fact is that it is explicitly or implicitly assumed to be zero. This characterizes secondorder circularity, which means that second-order statistics of $\mathbf{Z}$ and $\exp (j a) \mathbf{Z}$ arc the same for any $a$. This assumption of circularity [4] is sometimes even introduced in the definition, as, for example, in the normal case (sec [1, p. 431 and [5]). In [6], the term proper is used instead of "circular." However, circularity is only a particular assumption that is not always valid.

The question that immediately appears is to know whetheir the matrices $\boldsymbol{\Gamma}$ and $\mathbf{C}$ must only satisfy the conditions indicated above and deduced from their definition. The answer is no, and we shall establish a necessary and sufficient condition on the pair $(\boldsymbol{\Gamma}, \mathbf{C})$.

Proposition: Assuming that $\boldsymbol{\Gamma}$ is complex and positive definite and that $\mathbf{C}$ is complex and symmetric, this matrix $\mathbf{C}$ is a relation mahix of a random vector $\mathbf{Z}$ if and only if the matrix $\mathbf{C}^{*}-\mathbf{C}^{H} \boldsymbol{\Gamma}^{-1} \mathbf{C}$ is NND. 
Proof: Suppose first that $\mathbf{C}$ is the relation matrix of a RV Z. Consider now the RV W of $\mathbb{C}^{2 n}$ defined by $\left[\mathbf{Z}^{T}, \mathbf{Z}^{H}\right]$. Its covariance matrix is a $2 n \times 2 n$ complex matrix, and a simple calculation yields

$$
\boldsymbol{\Gamma}_{2}=\left(\begin{array}{cc}
\boldsymbol{\Gamma} & \mathbf{C} \\
\mathbf{C}^{*} & \boldsymbol{\Gamma}^{*}
\end{array}\right)=\left(\begin{array}{cc}
\boldsymbol{\Gamma} & \mathbf{C} \\
\mathbf{C}^{H} & \boldsymbol{\Gamma}^{*}
\end{array}\right) .
$$

As any covariance matrix, it is NND. Its Cholesky block factorization can be written as

$$
\boldsymbol{\Gamma}_{2}=\left(\begin{array}{cc}
\mathbf{I} & \mathbf{0} \\
\mathbf{R} & \mathbf{I}
\end{array}\right)\left(\begin{array}{cc}
\boldsymbol{\Gamma} & \mathbf{0} \\
\mathbf{0} & \mathbf{P}
\end{array}\right)\left(\begin{array}{cc}
\mathbf{I} & \mathbf{R}^{H} \\
\mathbf{0} & \mathbf{I}
\end{array}\right)
$$

where

$$
\mathbf{R}=\mathbf{C}^{H} \boldsymbol{\Gamma}^{-1} ; \mathbf{P}=\boldsymbol{\Gamma}^{*}-\mathbf{C}^{H} \boldsymbol{\Gamma}^{-1} \mathbf{C} .
$$

As $\boldsymbol{\Gamma}_{2}$ is NND, the diagonal-block matrix appearing in (3) is also NND. The fact that $\boldsymbol{\Gamma}$ is PD implies that $\mathbf{P}$ defined by (4) is NND, which gives the only if part.

Suppose now that $\mathbf{C}$ is such that $\mathbf{P}$ is NND. We have to show that there exists a complex RV $\mathbf{Z}$ satisfying (1). It results from (3) that if $\boldsymbol{\Gamma}$ is positive definite and $\mathbf{P}$ NND, then $\boldsymbol{\Gamma}_{2}$, which is defined by (2), is NND. This implies that there exists at least one RV of $\mathbb{C}^{2 n}$ such that its covariance matrix is $\Gamma$ (see [3, p. 651). However, this docs not mean that this RV can be partitioned as $\left[\mathbf{Z}^{T}, \mathbf{Z}^{H}\right]^{T}$ To arrive at this result, we must introduce the real and imaginary parts $\mathbf{X}$ and $\mathbf{Y}$. For this purpose, let $\boldsymbol{\Gamma}_{2 n}$ be the $2 n \times 2 n$ matrix defined by

$$
\boldsymbol{\Gamma}_{2 n}=\mathbf{M} \boldsymbol{\Gamma}_{2} \mathbf{M}^{H}
$$

where where $\mathbf{M}$ is defined by

$$
\mathbf{M}=\frac{1}{2}\left(\begin{array}{cc}
\mathbf{I} & \mathbf{I} \\
-j \mathbf{I} & j \mathbf{I}
\end{array}\right) \quad ; \quad \mathbf{M}^{-1}=\left(\begin{array}{cc}
\mathbf{I} & j \mathbf{I} \\
\mathbf{I} & -j \mathbf{I}
\end{array}\right) .
$$

It is clear that as $\boldsymbol{\Gamma}_{2}$ is NND, $\boldsymbol{\Gamma}_{2 n}$ is also NND. Furthermore, a simple calculation shows that $\boldsymbol{\Gamma}_{2 n}$ is a real symmetric matrix, or

$$
\boldsymbol{\Gamma}_{2 n}=\left(\begin{array}{cc}
\boldsymbol{\Gamma}_{x} & \boldsymbol{\Gamma}_{x y} \\
\boldsymbol{\Gamma}_{y x} & \boldsymbol{\Gamma}_{y}
\end{array}\right)
$$

with

$$
\begin{gathered}
\boldsymbol{\Gamma}_{x}=(1 / 2) \operatorname{Re}(\boldsymbol{\Gamma}+\mathbf{C}) ; \boldsymbol{\Gamma}_{x y}=(1 / 2) \operatorname{Im}(-\boldsymbol{\Gamma}+\mathbf{C}), \\
\boldsymbol{\Gamma}_{y x}=(1 / 2) \operatorname{Im}(\boldsymbol{\Gamma}+\mathbf{C}) ; \boldsymbol{\Gamma}_{y}=(1 / 2) \operatorname{Re}(\boldsymbol{\Gamma}-\mathbf{C}),
\end{gathered}
$$

where Re and Im stand for real and imaginary parts, respectively.

As $\boldsymbol{\Gamma}_{2 n}$ is symmetric and NND, it is possible to construct at least one vector of $\mathbb{R}^{2 n}$ written as $\left[\mathbf{X}^{T}, \mathbf{Y}^{T}\right]^{T}$ such that its covariance matrix is $\boldsymbol{\Gamma}_{2 n}$. Taking $\mathbf{Z}=\mathbf{X}+j \mathbf{Y}$, we easily obtain that the covariance and relation matrices of $\mathbf{X}$ are $\boldsymbol{\Gamma}$ and $\mathbf{C}$, respectively. This completes the proof. 


\section{NORMAL DISTRIBUTION}

Normal RV's arise in rnany areas of signal processing for wellknown reasons. In the complex case, it is almost always assumed that the RV's are also circular, which considerably simplifies the calculation (see [3, p. 1181. [SI, and [6]). We will present the situation appearing when circularity is not introduced.

A complex RV is said to be normal if its real if its real and imaginary parts $\mathbf{X}$ and $\mathbf{Y}$ are jointly normal. As a consequence, the PDF of such a vector with zero mean value is

$$
p(\mathbf{x}, \mathbf{y})=(2 \pi)^{-n}\left[\operatorname{det}\left(\boldsymbol{\Gamma}_{2 n}\right)\right]^{-1 / 2} \exp \left(-\frac{1}{2}\right) q(\mathbf{x}, \mathbf{y}),
$$

with

$$
q(\mathbf{x}, \mathbf{y})=\mathbf{v}^{T} \boldsymbol{\Gamma}_{2 n}^{-1} \mathbf{v}=\mathbf{v}^{H} \boldsymbol{\Gamma}_{2 n}^{-1} \mathbf{v}
$$

In this quadratic form, $\mathbf{v}$ is the vector of $\mathbb{R}^{2 n}$ defined by $\mathbf{v}^{T}=\left[\mathbf{x}^{T}, \mathbf{y}^{T}\right]$, and $\boldsymbol{\Gamma}_{2 n}$ is the covariance matrix defined by (7). It is a $2 n \times 2 n$ matrix, and the $n \times n$ matrices appearing in its block decomposition are

$$
\boldsymbol{\Gamma}_{x}=E\left(\mathbf{X X}^{T}\right) ; \boldsymbol{\Gamma}_{y}=E\left(\mathbf{Y} \mathbf{Y}^{T}\right) ; \boldsymbol{\Gamma}_{x y}=E\left(\mathbf{X Y}^{T}\right)
$$

Note that the last equality of (11) comes from the fact that all the previous elements are real.

As noted, for example, in [7] or [8], it is clear that $\mathbf{x}$ and $\mathbf{y}$ in (10) can be expressed in terms of $\mathbf{z}=\mathbf{x}+j \mathbf{y}$ or of $\mathbf{z}^{*}$, which introduces another form of the PDF. The calculation of this PDF is given in [7], and we present here a derivation giving the same result but expressed in terms of the matrices $\boldsymbol{\Gamma}$ and $\mathbf{C}$ previously introduced and not expliciily used in [7].

Let $\mathbf{w}$ be the vector defined by $\left[\mathbf{z}^{T}, \mathbf{z}^{H}\right]$ analog to the RV $\mathbf{W}$ introduced above. It results; from this definition that

$$
\mathbf{w}=\mathbf{M}^{-1} \mathbf{v} ; \mathbf{v}=\mathbf{M w}
$$

where is the matrix given by (6). As a consequence, the quadratic form (11) can be expresse'd as

$$
q(\mathbf{x}, \mathbf{y})=q^{\prime}\left(\mathbf{z}, \mathbf{z}^{*}\right)=\mathbf{w}^{H} \boldsymbol{\Gamma}_{w}^{-1} \mathbf{w}
$$

with $\boldsymbol{\Gamma}_{w}^{-1}=\mathbf{M}^{H} \boldsymbol{\Gamma}_{2 n}^{-1} \mathbf{M}$ Furthermore, the classical rule for the product of determinants yields $\operatorname{det} \boldsymbol{\Gamma}_{2 n}^{-1}=$ $\operatorname{det}\left(\boldsymbol{\Gamma}_{w}\right)|\operatorname{det}(\mathbf{M})|^{2}$. By using the fact that a determinant is unchanged by adding rows or columns, it results from (6) that $\operatorname{det}(\mathbf{M})=j^{n} 2^{-n}$, and therefore, $\left[\operatorname{det}\left(\boldsymbol{\Gamma}_{2 n}\right)\right]^{-1 / 2}=2^{n}\left[\operatorname{det}\left(\boldsymbol{\Gamma}_{w}\right)\right]^{-1 / 2}$. By combining all these results, we can express the PDF (10) as

$$
\begin{gathered}
p(\mathbf{x}, \mathbf{y})=p^{\prime}\left(\mathbf{z}, \mathbf{z}^{*}\right)= \\
\pi^{-n}\left[\operatorname{det}\left(\boldsymbol{\Gamma}_{w}\right)\right]^{-1 / 2} \exp \left(-\frac{1}{2} \mathbf{w}^{H} \boldsymbol{\Gamma}_{w}^{-1} \mathbf{w}\right) .
\end{gathered}
$$


This is the result given in [7]. Let us now calculate $\boldsymbol{\Gamma}_{w}$ appearing in (14) in terms of the elements of $\boldsymbol{\Gamma}_{2 n}$ defined by (12). By using (6), we obtain that $\boldsymbol{\Gamma}_{w}$ is the matnx $\boldsymbol{\Gamma}_{2}$ defined by (2), where

$$
\begin{gathered}
\boldsymbol{\Gamma}=\boldsymbol{\Gamma}_{x}+\boldsymbol{\Gamma}_{y}+j\left(\boldsymbol{\Gamma}_{y x}-\boldsymbol{\Gamma}_{x y}\right) \\
\boldsymbol{\Gamma}_{y x}=\boldsymbol{\Gamma}_{x}-\boldsymbol{\Gamma}_{y}+j\left(\boldsymbol{\Gamma}_{y x}+\boldsymbol{\Gamma}_{x y}\right)
\end{gathered}
$$

These equations are, of course, equivalent to (8) and (9). By using (3) for the determinant and by combining all these results, (15) takes the form

$$
\begin{gathered}
p(\mathbf{x}, \mathbf{y})=p^{\prime}\left(\mathbf{z}, \mathbf{z}^{*}\right)= \\
\pi^{-n}[\operatorname{det}(\mathbf{\Gamma}) \operatorname{det}(\mathbf{P})]^{-1 / 2} \exp \left[-\frac{1}{2} q^{\prime}\left(\mathbf{z}, \mathbf{z}^{*}\right)\right],
\end{gathered}
$$

with

$$
q^{\prime}\left(\mathbf{z}, \mathbf{z}^{*}\right)=\left[\mathbf{z}^{H}, \mathbf{z}^{\mathbf{T}}\right]\left(\begin{array}{cc}
\boldsymbol{\Gamma} & \mathbf{C} \\
\mathbf{C}^{H} & \boldsymbol{\Gamma}^{*}
\end{array}\right)\left(\begin{array}{c}
\mathbf{z} \\
\mathbf{z}^{*}
\end{array}\right)
$$

The principal interest of this expression is the fact that it uses only the two matrices $\mathbf{\Gamma}$ and $\mathbf{C}$ defined by (1) and having a simple meaning in terms of the complex random vecto $\mathbf{Z}$

There is a case that is especially important. It appears when $\mathbf{C}=\mathbf{0}$, which means that the random vector $\mathbf{Z}$ is circular. With this property, the previous equations become

$$
p(\mathbf{x}, \mathbf{y})=p_{C}(\mathbf{z})=\pi^{-n}[\operatorname{det}(\boldsymbol{\Gamma})]^{-1} \exp \left[-\mathbf{z}^{H} \boldsymbol{\Gamma}^{-1} \mathbf{z}\right],
$$

which is the classical expression of the PDF of a circular normal vector.

It is now interesting to explicitly express the matrix, appearing in the quadratic form (18). By using a simple inverse calculation, we obtain

$$
\left(\begin{array}{cc}
\boldsymbol{\Gamma} & \mathbf{C} \\
\mathbf{C}^{H} & \boldsymbol{\Gamma}^{*}
\end{array}\right)=\left(\begin{array}{cc}
\mathbf{P}^{-*} & -\mathbf{R}^{H} \mathbf{P}^{-1} \\
-\mathbf{R}^{T} \mathbf{P}^{-*} & \mathbf{P}^{-1}
\end{array}\right)
$$

where the matrices $\mathbf{P}$ and $\mathbf{R}$ are defined by (4), and $\mathbf{P}^{-*}$ means $\left(\mathbf{P}^{-1}\right)^{*}$. With this matrix, the quadratic form (18) becomes

$$
q^{\cdot}\left(\mathbf{z}, \mathbf{z}^{*}\right)=2\left[\mathbf{z}^{H} \mathbf{P}^{-*} \mathbf{z}-\operatorname{Re}\left(\mathbf{z}^{T} \mathbf{R}^{T} \mathbf{P}^{-*} \mathbf{z}\right)\right]
$$

Finally, the PDF (17) can be written as

$$
p\left(\mathbf{z}, \mathbf{z}^{*}\right)=\pi^{-n}[\operatorname{det}(\boldsymbol{\Gamma}) \operatorname{det}(\mathbf{P})]^{-1 / 2} \exp \left[-\mathbf{z}^{H} \mathbf{P}^{-*} \mathbf{z}-\operatorname{Re}\left(\mathbf{z}^{T} \mathbf{R}^{T} \mathbf{P}^{-*} \mathbf{z}\right)\right]
$$

It is possible to put this expression in another form. Applying the matrix inversion lemma (see [I, p. 241) to the matrix $\mathbf{P}^{-*}$ given by (4) yields

$$
\mathbf{P}^{-*}=\boldsymbol{\Gamma}^{-1}+\boldsymbol{\Gamma}^{-1} \mathbf{C P}^{-1} \mathbf{C}^{H} \boldsymbol{\Gamma}^{-1} .
$$


By inserting this expression in (22), the circular PDF $p_{C}(\mathbf{Z})$ defined by (19) appears, and this gives

$$
p\left(\mathbf{z}, \mathbf{z}^{*}\right)=p_{C}(\mathbf{x})\left[\operatorname{det}\left(\boldsymbol{\Gamma}^{-1} \mathbf{P}\right]^{-1 / 2} \exp \left[-\mathbf{z}^{H} \mathbf{R}^{H} \mathbf{P}^{-1} \mathbf{R} \mathbf{z}+\operatorname{Re}\left(\mathbf{z}^{T} \mathbf{R}^{T} \mathbf{P}^{-*} \mathbf{z}\right)\right]\right.
$$

Finally, it can be noted that as $\mathbf{P}$ is Hermitian and positive $\operatorname{definite} \operatorname{det}(\mathbf{P})=\operatorname{det}\left(\mathbf{P}^{*}\right)$, and by using (4), we obtain $\operatorname{det}\left(\boldsymbol{\Gamma}^{-1} \mathbf{P}\right)=\operatorname{det}\left(\mathbf{I}-\mathbf{R}^{H} \mathbf{R}^{T}\right)$. As a result, the most general PDF of a complex normal $\mathrm{RV}$ can be factorized in a product of the PDF corresponding to the circular case by a function depending only on the matrices $\mathbf{R}$ and $\mathbf{P}$ defined by (4). A similar result is used in [8].

It is clear thatt the same procdur can be applied for the calcultion of the characteristic function of comples normal random vectors which is more convefntneyt than the PDF in various calculations.

\section{REFERENCES}

[1] S. Kay, Modern Spectral Analysis, Theory and Applications., Englewood Cliffs, N.J. : Prentice Hall, 1989.

[2] R. A. Monzigo and T. Vv. Miller, Introduction to AdaptiveArray.s., New-York: Wiley, 1980.

[3] B. Picinbono, Random Signals and Systems, Englewood Cliffs, N.J. : Prentice Hall, 1993.

[4] B. Picinbono, “On circularity," IEEE Trans. Signal Processing, vol. 42, pp. 3473-3482, 1994.

[5] K. Miller, Multidimensional Gaussian Distributions. New York: Wiley, 1964.

[6] F. Neeser and J. Massey, "Proper complex random procesires with applications to information theory." IEEE Trans. Inform. Theory, vol. 39, pp, 1293-1302, 1993.

[7] A. van den Bos, "The: multivariate complex normal distribution-A generalization," IEEE Trans. Inform. Theory, vol. 41, pp. 537-539, 1995.

[8] P. Amblard and P. Duvaut, "Filtrage adapt6 dans le cas gaussien complexe non circulaire," in Proc. 15th Symp. GRETSI,, 1995, pp. 141-144.

[9] B. Picinbono and P. Chevalier, "Widely linear estimation with complex data." IEEE Trans. Signal Processing, vol. 43, pp. 2030-2033, 1995. 